\title{
Performance Test Comparison of Internet Layer Protocols on CentOS and 2007 for Data, DNS and Voice Traffic
}

\author{
Shubhangi Kharche \\ Assistant Professor, EXTC Department \\ SIES Graduate School of Technology \\ Nerul, Navi Mumbai
}

\author{
Pramod B. Patil, PhD. \\ Professor \& Principal \\ Fellow IETE, LMISTE, \\ J D College of Engineering, Nagpur.
}

\begin{abstract}
Today's era of packet switched networks demands larger bandwidth to suffice the need to integrate multimedia applications like Internet gaming, transmission of voice etc. It becomes necessary to judge the network performance with the allocated bandwidth. Network performance depends mainly on the efficiency of the protocol used in addition to load on the network, the transmission system type and the connected hardware capabilities. The performance of the two versions of Internet Protocol IPv4 and IPv6 is tested as well as compared on CentOS and windows 2007 operating systems for different voice samples, DNS traffic, data traffic and Internet gaming traffic characteristics like counterstrike and Quake III. The transport layer data traffic and the application layer DNS and voice traffic was generated using the latest version of Distributed Internet Traffic Grapher tool; D-ITG 2.8.0 rc1.The effect of transmitting voice over IP with compressed RTP and with and without voice activity detection is also observed.
\end{abstract}

\section{Keywords}

2007, CentOS, CODEC, Crtp, D-ITG 2.8.0 rc1, Internet Layer Protocols,VAD,VoIP

\section{INTRODUCTION}

Version 4 of Internet Protocol has worked well to its maximum capacity since the birth of the Internet. Due to its shortcomings like limited addressing capability, poor Quality of service ,security and mobility issues to name a few it is gradually been replaced by higher version 6 of Internet Protocol.16 byte IPv6 address can identify $10^{28}$ more hosts in Internet as compared to 4 million hosts identified by 4 byte IPv4 address[1]. Today the Internet has grown to a stage of even interconnecting multiples of millions of small things with the support of IPv6.It is not affordable to discard the existing IPv4 network infrastructure, so several mechanisms have been implemented for the transition to IPv6 networks .In view of the coexistence of IPv4 and IPv6 it becomes necessary to test their performance. In this paper we measured and compared the performance of versions 4 and 6 of Internet Protocols on the latest operating system CentOS and windows 2007 in peer to peer environments using throughput as the primary metric. We also tested their performance with jitter measurements since it is an important measure when voice is transmitted through Internet.
The paper is structured to explain transmission of IP packets, transmission of Voice over IP, DNS and Internet gaming in section 2. Network test platform is explained in section 3 . Performance tests results and discussion is covered in section 4 followed by conclusion in section 5 .

\section{COMMUNICATION WITH IP}

\subsection{Transmission of IP packets}

Internet Protocol(IP) the network layer protocol of TCP/IP model is connectionless and carries 32 bit address for version 4 (or 128 bit address for version 6) in its header to ensure host to host delivery of packets.However no field of IP header can identify the process from which the message is sent. Different processes running on an application are identified by port numbers which are a part of TCP and UDP protocol headers at transport layer [2, 3].So IP Packet is always transmitted along with TCP and UDP. The TCP header is 20 bytes, UDP header is 8 bytes, IPv4 header is 20 bytes and IPv6 header is 40 bytes (fixed) or 60 bytes (with options). IP packet is composed of IP header and data. Therefore,

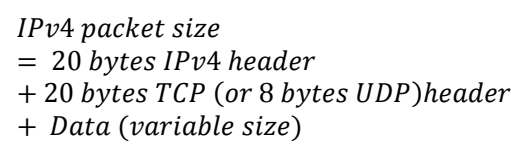

Maximum IPv4 data size

$$
\begin{aligned}
& =2^{16} \\
& -20 \text { bytesfixedheader } \\
& =65516 \text { bytes }
\end{aligned}
$$

Thus when IPv4 packet is transmitted using TCP,

$$
\begin{aligned}
\text { Maximum TCP } / I P v 4 & \text { data size } \\
& =65516 \\
& -20 \text { bytes TCP header } \\
& =65496 \text { bytes }
\end{aligned}
$$

And when IPv4 packet is transmitted using UDP,

$$
\begin{aligned}
\text { Maximum UDP/IPv4 data size } & \\
& =65516 \\
& -8 \text { bytes UDP header } \\
& =65508 \text { bytes }
\end{aligned}
$$




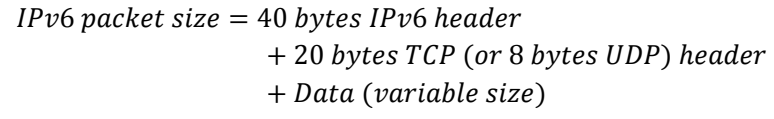

MaximumIPv6 datasize $=2^{16}=65536$ bytes (Since the payload length in IPv6 header does not include the header length). Thus when IPv6 packet is transmitted using TCP,

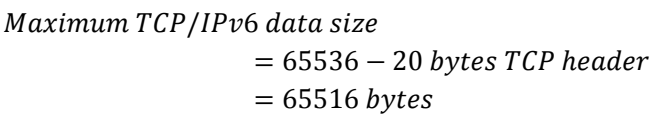

And when IPv6 packet is transmitted using UDP,

Maximum UDP/IPv6 data size

$$
\begin{aligned}
& =65536-8 \text { bytes UDP header } \\
& =65528 \text { bytes }
\end{aligned}
$$

One of the strongest features of IP is that it works with any underlying physical layer technology. When IP packets are transmitted over Ethernet they are encapsulated in a frame with a minimum and maximum length of 64 bytes and 1518 bytes respectively. The Ethernet frame has 14 bytes header and 4 bytes trailer. Therefore minimum size of IP packet that can travel through Ethernet is 46 bytes (64bytes-14bytes- 4 bytes) and its maximum size is 1500 bytes (1518bytes14bytes-4bytes) [4].when less than 46 bytes IP packet is transmitted options field of its header is used to pad the extra bytes required and when more than 1500 bytes IP packet is transmitted fragmentation and reassembly [5] is done by IP. This fragmentation and reassembly increases processing overhead thereby reducing the network speed.

\subsection{Transmission of Voice over IP (VoIP)}

CODEC (Compressor and Decompressor) is required to convert the analogue voice into digital before transmitting it through IP (Internet Protocol) networks [6]. Every conversation is an amalgam of voice period and silence period. In order to save bandwidth VAD (voice activity detection) is used which aids in the transmission of only voice by isolating the silence. Real time voice is always carried by RTP (Real Time Protocol) over UDP (User Datagram Protocol) [7].Thus when voice is transmitted over IP;

$$
\begin{aligned}
\text { VoIPv4 headersize } & =12 \text { bytes RTP header } \\
& +8 \text { bytes UDP header } \\
& +20 \text { bytes IPv } 4 \text { header } \\
& =40 \text { bytes }
\end{aligned}
$$

VoIPv6 header size $=12$ bytes $R T P$ header

$$
\begin{aligned}
& +8 \text { bytes UDP header } \\
& +40 \text { bytes IPv } 6 \text { header } \\
& =60 \text { bytes }
\end{aligned}
$$

VoIPv4/6 packet size $=40 / 60$ bytes header

$$
+ \text { payload }
$$

$$
\text { (variable size depending on codec) }
$$

If 40 bytes voice sample is transmitted then overhead caused due to IPv4 is $50 \%(40 / 80)$ and that caused due to IPv6 is $60 \%(60 / 100)$ thereby resulting in bandwidth wastage. To avoid the latter Compressed RTP (Crtp) is used which reduces the headers to two or four bytes $[8,9]$.

\subsection{DNS and Internet gaming}

Applications running on Internet require Domain Name System (DNS) to map the difficult to remember lengthy numeric IP addresses with the user friendly names. Gaming applications like First Person Shooter (FPS) on Internet are characterized by counterstrike and Quake3 traffic [10]. Since Internet games contribute to maximum traffic nowadays throughput tests are also carried for the aforesaid traffic characteristics.

\section{NETWORK TEST PLATFORM}

We carried our performance tests on two computers with similar hardware specifications and connected in fast Ethernet via category 6 crossover cable as seen in figure1.Both the computers were dual booted with CentOS and windows 2007.We connected the two computers with a point to point link to avoid degradation in the network speed due to the effects of processing overheads at the intermediate nodes.

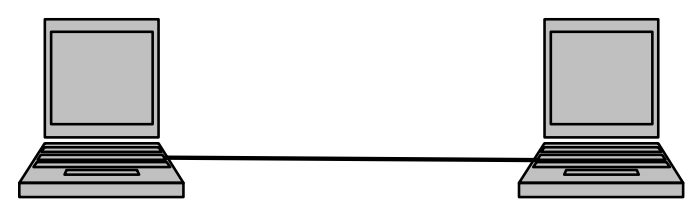

$$
\begin{aligned}
& \text { Computer } 1 \\
& \text { IPv4 } \rightarrow \text { 10.10.10.4 } \\
& \text { IPv6 } \rightarrow \text { 1234:470:e1ce: } 1:: 100
\end{aligned}
$$

$$
\begin{aligned}
& \text { Computer } 2 \\
& \text { IPv4 } \rightarrow \text { 10.10.10.11 } \\
& \text { IPv6 } \rightarrow \text { 1234:470:e1ce:1::101 }
\end{aligned}
$$

\section{Figure1: Network Test Platform}

The computers were first booted with CentOS; their IP stacks were configured for version 4 and then were tested for connectivity by using ping utility for IPv4 address. We got a successful ping reply for the default 56 bytes of ICMP data as seen in figure2.We then carried the performance tests for IPv4 on CentOS.

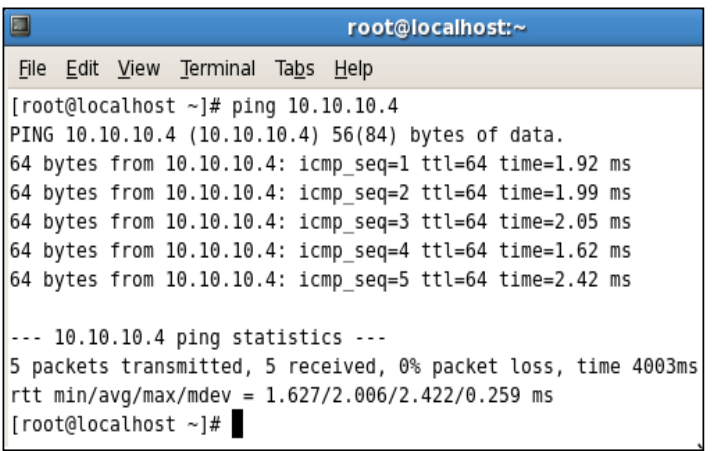

\section{Figure2: IPv4 Ping results for CentOS}

We then used ping6 utility for IPv6 address and got the successful reply again with the default 56 bytes of ICMP data as seen in figure 3.After this connectivity confirmation reply we carried the performance tests for IPv6 on CentOS. Then 
the computers were booted with windows 2007 and the similar performance tests were repeated.

\subsection{Traffic Generation Tool}

D-ITG 2.8.0rc1 [11] generates traffic at the higher three layers of the Internet and measures performance metrics like throughput, jitter, and delay.

\subsection{Throughput}

It is measured in terms of the number of bits, bytes or packets transmitted over a specified amount of time in seconds. It is an important metric as it depicts how fast data can be transmitted. The equation below gives the relation between throughput in bits per second and packets per sec.

\section{root@localhost:}

\section{File Edit View Terminal Tabs $\underline{\text { Help }}$}

[root@localhost ]\# ping6 1234:470:elce:1::101

PING 1234:470:elce:1::101(1234:470:elce:1::101) 56 data bytes

64 bytes from 1234:470:elce:1::101: icmp_seq=0 ttl=64 time $=28.4 \mathrm{~ms}$ 64 bytes from 1234:470:elce:1::101: icmp_seq $=1$ ttl $=64$ time $=2.80 \mathrm{~ms}$ 64 bytes from 1234:470:elce:1::101: icmp_seq $=2$ ttl $=64$ time $=2.22 \mathrm{~ms}$ 64 bytes from 1234:470:elce:1::101: icmp_seq $=3$ ttl $=64$ time $=2.16 \mathrm{~ms}$ 64 bytes from 1234:470:elce:1::101: icmp_seq $=4$ ttl $=64$ time $=2.06 \mathrm{~ms}$ 64 bytes from 1234:470:elce:1::101: icmp_seq=5 ttl=64 time $=2.09 \mathrm{~ms}$ 64 bytes from 1234:470:elce:1::101: icmp_seq $=6$ ttl $=64$ time $=2.75 \mathrm{~ms}$

Figure3: IPv6 Ping results for CentOS

\subsection{Traffic Generation Tool}

D-ITG 2.8.0rc1 [11] generates traffic at the higher three layers of the Internet and measures performance metrics like throughput, jitter, and delay.

\subsection{Throughput}

It is measured in terms of the number of bits, bytes or packets transmitted over a specified amount of time in seconds. It is an important metric as it depicts how fast data can be transmitted. The equation below gives the relationbetween throughput in bits per second and packets per sec.

\section{(Throughput)bps $=($ Packetspersecond $)$ \\ $*($ packetsizeinbytes $) * 8$ bits}

\subsection{Jitter}

It is varied delay in milliseconds and an important measure when voice is being transmitted over IP.

\section{PERFORMANCE TEST RESULTS AND DISCUSSION}

D-ITG 2.8.0rc1 tool was installed on both the computers. The round trip time meter was set for constant trafficdistribution on the sender machine. We first enabledthe IPv4 stack and had run the tool for 15 times for theperformance tests to avoid any error in the measurements and then averaged all the results. Before running the tool we decided the range of data bytes for TCP and UDP from 32 bytes to 1408 bytes which would satisfy the Ethernet MTU and would avoid the use of options field of IP header. Further we varied the data bytes from 1536 bytes to 65496 bytes in order to analyze the effects of fragmentation and reassembly. After completing the measurements for IPv4 we enabled the IPv6 and repeated the same tests.

\subsection{TCP/IPv4 and TCP/IPv6 Throughput comparison on windows 2007 and CentOS.}

In this section we present the IPv4 and IPv6 throughput results when transmission is done using TCP.Figure4 below shows the measured TCP/IPv4 and TCP/IPv6 throughput in megabits per second (Mbps) for data bytes varying from 32 bytes to 65496 bytes. As seen in figure 4 both the operating systems perform similar for lower size of data bytes till 1152 bytes. Less significant variation is seen from 1280 bytes to 1792 bytes. At 1920 bytes and 2048bytes variations are least significant. However the throughput variation between the operating systems is large from 4096 to 65496 bytes with a maximum difference of $165.38 \%$ for TCP/IPv6 and $192.94 \%$ for TCP/IPv4 at 8192 bytes. As seen windows 2007 compared to CentOS performs well for higher size of data bytes.Moreover for data bytes beyond the MTUof Ethernet, TCP/ IPv6 throughput degrades compared to that of TCP/IPv4 by a maximum of $40.29 \%$ on CentOS and $30.48 \%$ on windows 2007 due to the effect of fragmentation and reassembly.

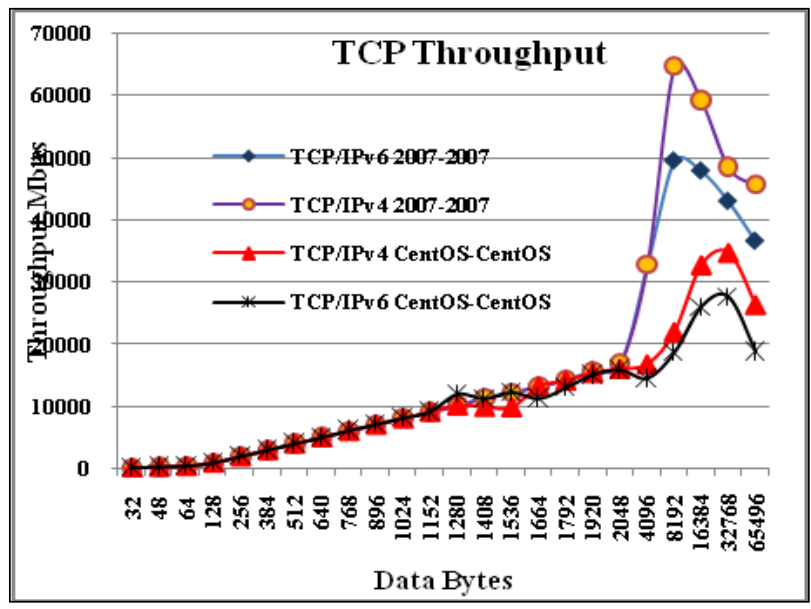

Figure 4: TCP throughput comparison betweenthe two IP versions for CentOS and windows 2007.

\subsection{UDP/IPv4 and UDP/IPv6 Throughput comparison on windows 2007 and CentOS.}

As seen in figure 5, for lower size of data bytes from 32 to 768 bytes the UDP throughput differences between windows 2007 and CentOS are neglible. However the differences increase at 896 bytes, 1280, 1536 and 1664 bytes and are significant. For higher data bytes from 4096 to 65496 bytes the differences are remarkably large and highest of $187.16 \%$ for IPv6 and 233.34\% for IPv4 at 65496 bytes. As seen again windows 2007 compared to CentOS performs well for higher size of data bytes. Moreover for 
data bytes beyond the MTU of Ethernet, UDP/ IPv6 throughput degrades compared to that of UDP/IPv4 by a maximum of $38.5 \%$ on CentOS and $17.77 \%$ on windows 2007 due to the effect of fragmentation and reassembly.

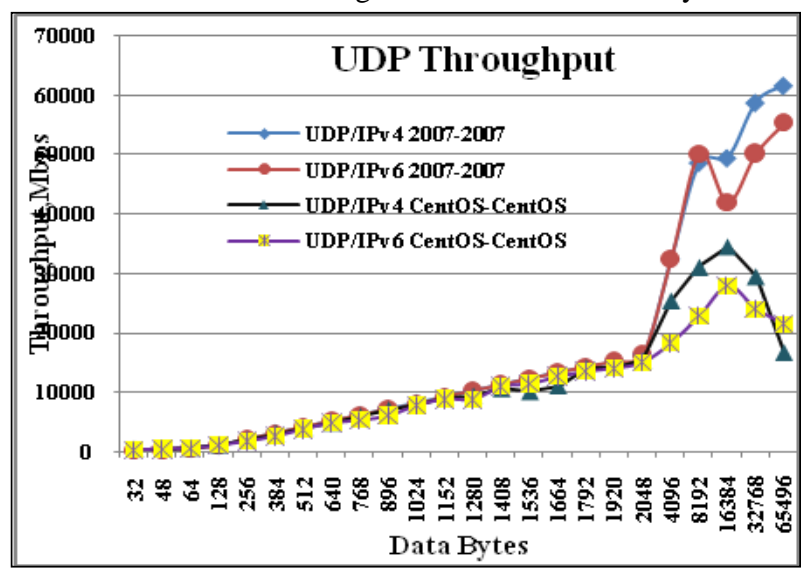

Figure 5: UDP throughput comparison between the two IP versions for CentOS and windows 2007.

\subsection{Overhead comparison for both versions of IP over TCP and UDP traffic}

Protocol Overhead is expressed in percentage and it is the ratio of the header size in bytes to the total bytes in the data unit. Thus for IP,

$$
\% \text { Overhead }=\frac{\text { HeaderSize }}{\text { TotalBytesinanIPpacket }} \times 100
$$

Table1:TCP/IP and UDP/ IP Overheads for both versions of IP.

\begin{tabular}{|l|l|l|l|l|}
\hline & TCP/ & TCP/ & UDP/ & UDP/ \\
IPv4 & IPv6 & IPv4 & IPv6 \\
\hline $\begin{array}{l}\text { Overhead } \\
\text { for 32 bytes } \\
\text { of data }\end{array}$ & $55.55 \%$ & $65.21 \%$ & $46.67 \%$ & $60 \%$ \\
\hline
\end{tabular}

Table1 depicts the IPv4 and IPv6 overheads for 32 bytes of data for both transport layer protocols. It is seen that the TCP overheads are higher for both versions of IP than the corresponding UDP overheads due to the 20 bytes TCP header as opposed to 8 bytes UDP header.

Figure6 depicts that as the data bytes size increases the percentage overhead for both versions of IP over both TCP and UDP decreases. Due to fragmentation at higher data bytes beyond 1500 bytes more headers are created which leads to increase in overhead and hence IPv6 throughput is significantly less than that of IPv4 for both TCP and UDP.

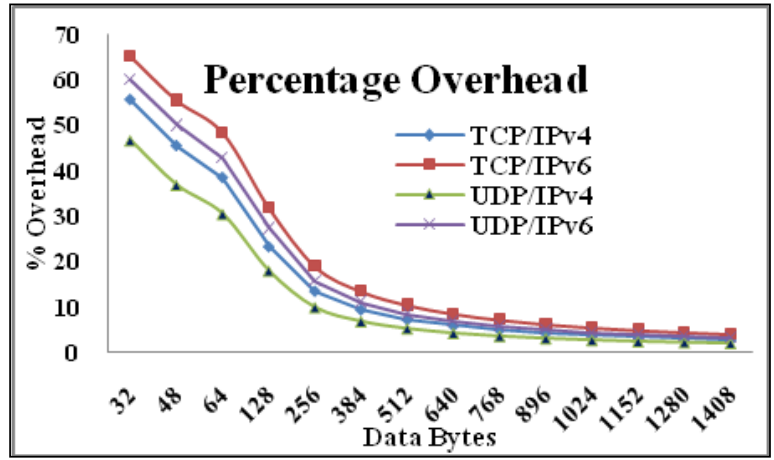

Figure6:TCP/IP and UDP/ IP Overheads for both versions of IP versus data bytes size

\subsection{TCP/IPv4 and TCP/IPv6 Jitter comparison on windows 2007 and CentOS}

It is seen from figure7 that CentOS performs well compared to windows 2007 in terms of TCP/IP Jitter. The jitter values on CentOS vary from $0.0007 \mathrm{sec}$ to $0.02 \mathrm{sec}$ whereas on windows 2007 they vary from $190.1 \mathrm{sec}$ to $1525.94 \mathrm{sec}$. Much difference is not seen between IPv4 and IPv6 jitter values on 2007.However considerable jitter differences between IPv4 and IPv6 are seen at certain data bytes on CentOS, maximum jitter difference being $106.02 \%$ at 32768 bytes.

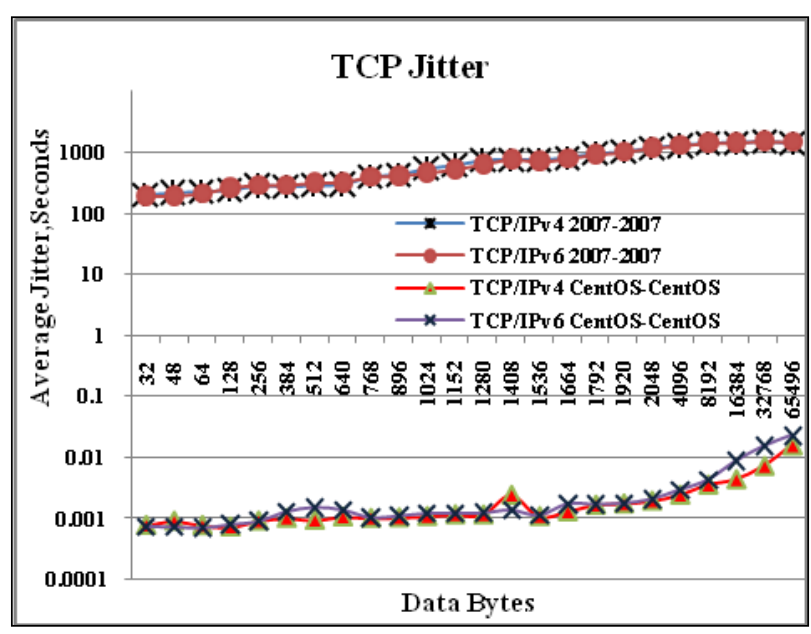

Figure7: TCP Jitter comparison between the two IP versions for CentOS and windows 2007.

\subsection{UDP/IPv4 and UDP/IPv6 Jitter}

\section{comparison on windows 2007 and CentOS}

It is seen from figure8 that Centos performs well as compared to win 2007 in terms of UDP jitter. The jitter values on CentOS vary from 0.0008 seconds to 0.02 seconds. Whereas on windows 2007 they vary from 281.34 seconds to 1409.93 seconds.On CentOS the maximum difference between IPv4 and IPv6 jitter is observed at 1664 bytesIPv4 has overall better performance than IPv6 for different data bytes. On windows 2007 the performances of IPv4 and IPv6 slightly vary with a maximum jitter difference of $11.01 \%$ at 32 bytes of data. 


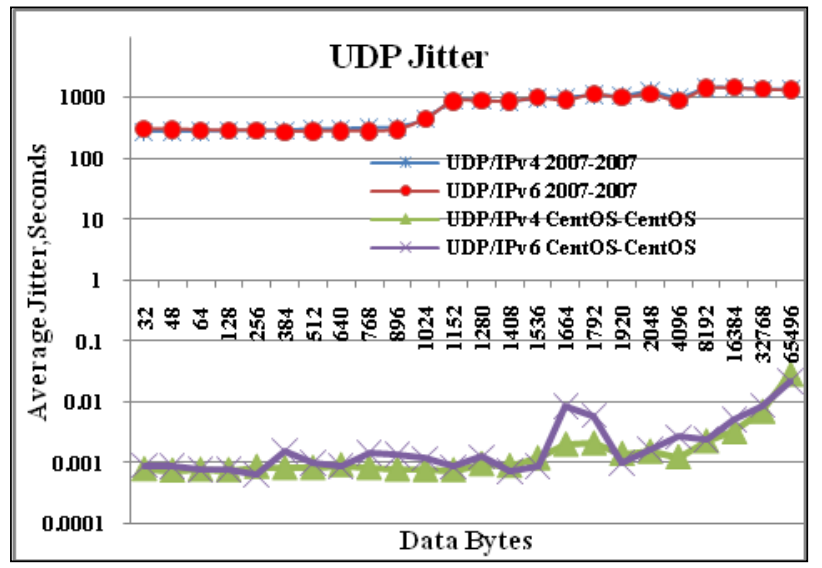

Figure 8: UDP Jitter comparison between the two IP versions for CentOS and windows 2007

\subsection{DNS Throughput comparison on Windows 2007 and CentOS.}

Figure9 depicts that CentOS performs well for DNS-TCP traffic over IPv4 as compared to that with windows 2007.However the later performs well for DNS-TCP traffic over IPv6 as compared to that with CentOS. Moreover on CentOS DNS-TCP throughput for IPv6 is at a lower side by $7.5 \%$ than that for IPv4 and on windows 2007 it is at a lower side by $2.04 \%$ than that for IPv4.

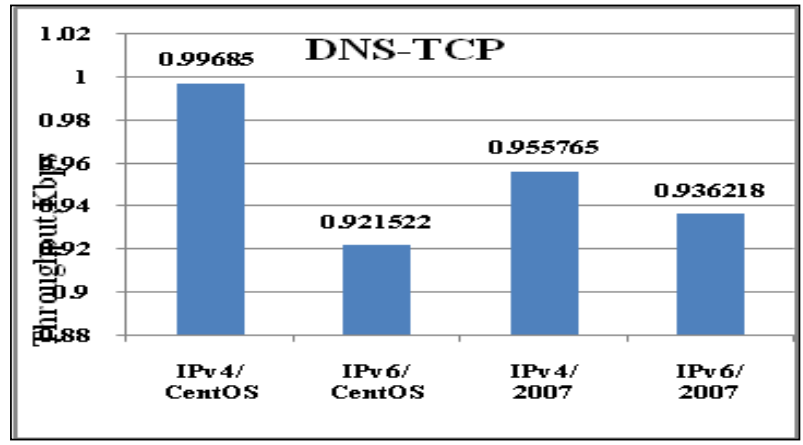

Figure 9: DNS-TCP Throughput comparison between the two IP versions for CentOS and windows 2007.

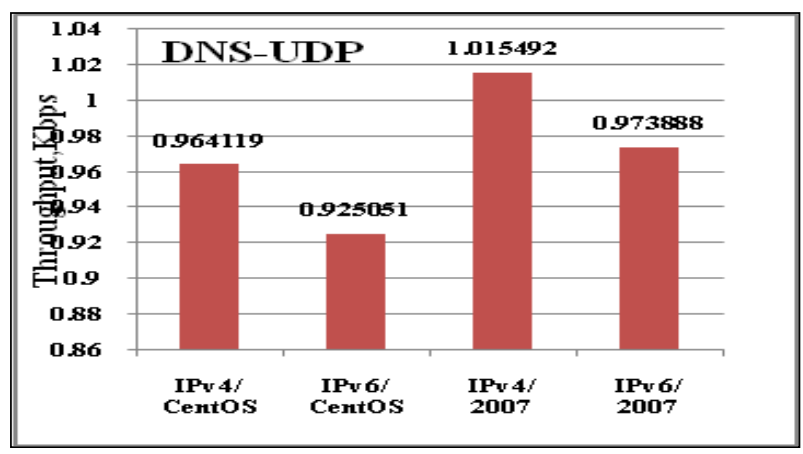

Figure10: DNS-UDP Throughput comparison between the two IP versions for CentOS and windows 2007.

It is clear from figure10 that windows 2007 performs well for DNS-UDP traffic over both IPv4 and IPv6 as correspondingly compared to Centos. The IPv6 throughput for DNS-UDP is at a lower side than that of IPv4 by $4.05 \%$ and $4.09 \%$ on CentOS and 2007 respectively.

\subsection{Throughput comparison for Internet gaming traffic characteristics on windows 2007 and CentOS.}

As seen from figure11 below, for all the three traffic characteristics IPv6 throughput is slightly better than that of IPv4 on both operating systems. The maximum throughput difference is $1.26 \%$ for Quake 3 characteristics on CentOS and $12.5 \%$ for counter strike inactive characteristics on windows 2007.

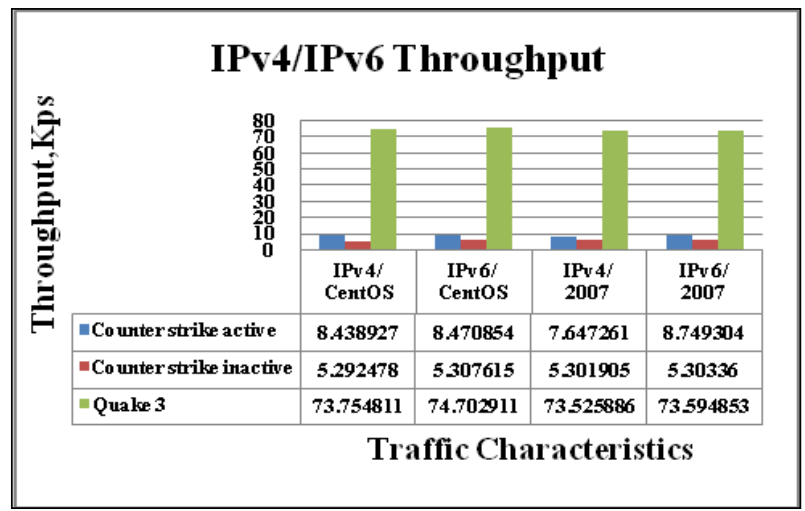

Figure11:IPv4/IPv6 Throughput comparison for different traffic characteristics on CentOS and windows 2007

\subsection{Throughput comparison for different voice samples on windows 2007 and CentOS.}

Figure12 shows that with VAD the IPv4/IPv6 throughput differences are negligible for the five different toll quality [12] voice codecs on the two operating systems. However the IPv6 throughput is slightly at a higher side on CentOS for all five voice codecs and on windows 2007 it is at a lower side only for G711.2.For G711.1, G723.1 and G729.2 it is same as that of IPv4 and for G729.3 it is slightly higher by $0.001 \%$ than that of IPv4. It is seen from figure 13 that the throughput performance for voice with Crtp on both operating systems is more or less similar. On CentOS the IPv6 throughput is slightly higher than that of IPv4 for all five voice codecs with a maximum difference of $0.006 \%$ for G729.2 codec. On windows 2007 IPv6 throughput is similar to that of IPv4 for G711.1, G723.1 and G729.2.However it is higher by $0.0016 \%$ for G711.2 and lowers by $0.0017 \%$ for G729.3 codec. Figure 14 depicts that the throughput performance for VAD Crtp is similar on both operating systems. However there are slight throughput differences between IPv4 and IPv6 on both operating systems for five different voice codecs. On CentOS IPv6 throughput is higher than that of IPv4 for all voice codecs except G729.2.The IPv4 throughput degrades by $6.24 \%$ for G729.2 on CentOS. Whereas on windows 2007 IPv6 throughput is higher by $0.00167 \%$ both for G723.1 and G729.3 .However it is similar to that of IPv4 for remaining three codecs G711.1, G711.2 and G729.2. 


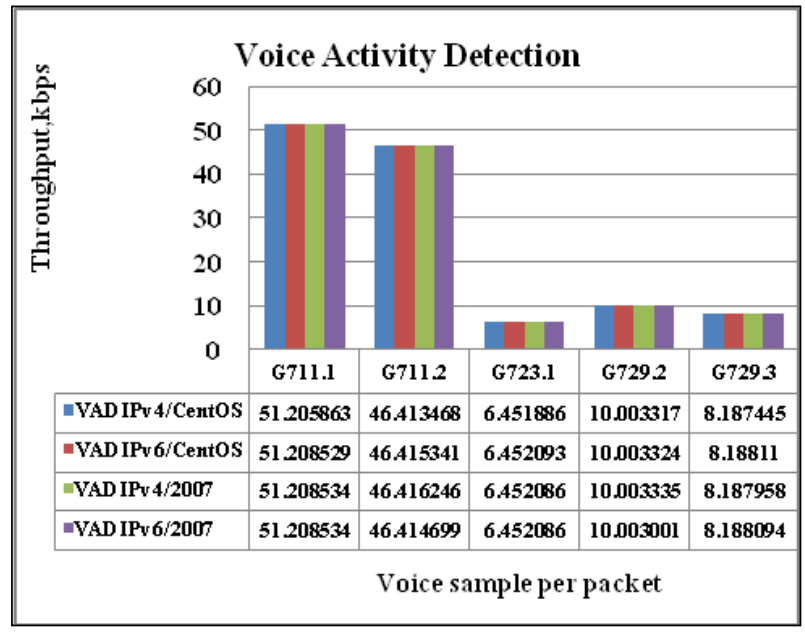

Figure12:IPv4/IPv6 Throughput comparison for different voice codecs with VAD on CentOS and windows 2007

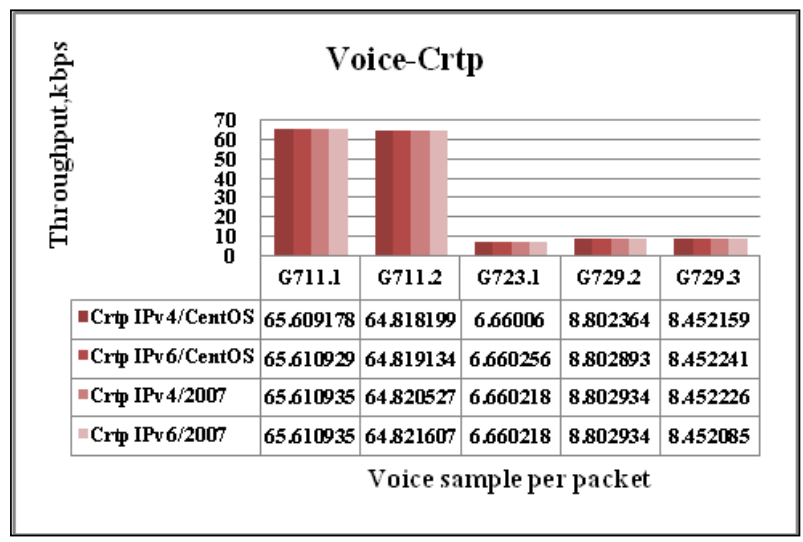

Figure 13:IPv4/IPv6 Throughput comparison for different voice codecs with VAD-Crtp on CentOS and windows 2007.

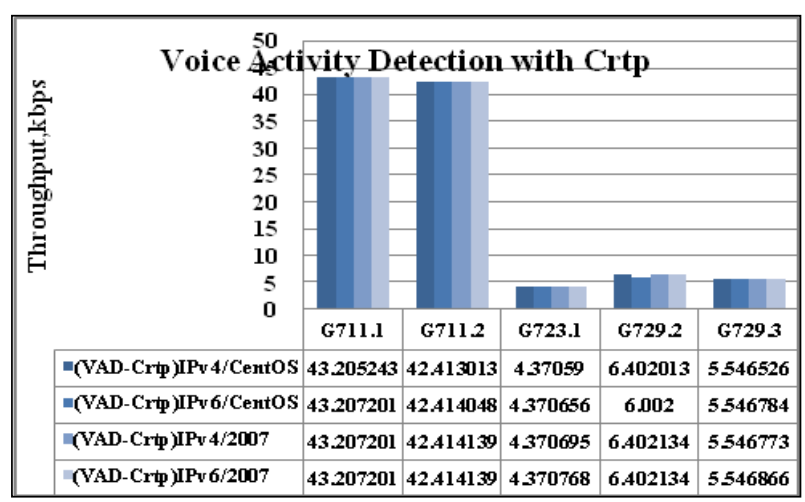

Figure 14:IPv4/IPv6 Throughput comparison for different voice codecs with VAD on CentOS and windows 2007

\section{CONCLUSION}

Following are the implications of the results:

- Windows 2007 performs well as compared to CentOS in terms of both TCP and UDP throughput at certain data bytes.
- CentOS performs well as compared to windows 2007 in terms of both TCP and UDP jitter at certain data bytes.

- On both operating systems IPv6 shows lower throughput performance as compared to that of IPv4 for higher size of data bytes due to its higher overhead.

- On both operating systems TCP shows lower throughput performance as compared to that of UDP for higher size of data bytes due to its higher overhead.

- Larger the data bytes size lesser is the percentage overhead but more is the overhead due to fragmentation and reassembly.

- However on both operating systems TCP shows lower jitter performance as compared to that of UDP for higher size of data bytes.

- IPv4 has slightly higher throughput performance than that of IPv6 for both DNS-TCP and DNS-UDP on both operating systems.

- Throughput performance for Quake3 traffic characteristics is highest among the three traffic characteristics. Throughput of counter strike traffic characteristics during the active phase of the game is better by maximum of $64.15 \%$ than that during that during the inactive phase of the game.

- Finally with voice activity detection bandwidth is saved but throughput is decreased as compared to the theoretical values for different voice codecs. Whereas in case of voice transmission with Crtp the theoretical and practical values of throughput are almost similar.

\section{REFERENCES}

[1] S.Deering, R.Hinden, 'Internet Protocol version 6 specifications' December 1998.

[2] Postel,J, 'Transmission control protocol'RFC 761,January 1998,www.ietf.org

[3] Postel,J, 'User Datagram protocol'RFC 761,August1980,www.ietf.org

[4] Jed Kaplan, 'Ethernet frame format', tools.ietf.org/html/draft-ietf-isis-ext-eth-01.

[5] A. Malis, 'Fragmentation and reassembly' ,tools.ietf.org/html/rfc4623

[6] M.Kondoz, 'Digital Speech'Coding for low bit rate communication systems, second edition, Wiley India.

[7] David J. Wright, 'Voice Over Packet Networks',Wiley Series on Communications technology,pp 76-84

[8] Dimitriadis,G.; Pavlidou,N. ;'Evaluation of Header Compression for VoIP Traffic over DVB-RCS', 16th IST Summit on Mobile and Wireless Communications, 2007 , pp $1-5$.

[9] Jin, H. ;Hsu, R. ; Jun Wang, 'Performance comparison of header compression schemes for RTP/UDP/IP packets' Wireless Communications and Networking Conference, WCNC.2004 IEEE, 21-25 March 2004, Vol.3,pp 1691 1696.

[10] Cricenti,A.L;Branch P.A;Armitage,G.J.'Time-series modelling of server to client IP packet Length in First person shooter games' $15^{\text {th }}$ IEEE conference on Networks ICON 2007,pp507-512.

[11] http://www.grid.unina.it/software/ITG/download.php/DITG 2.8.0- rc1 winbinary.

[12] Olivier Hersent,Jean-Pierre Petit,David Gurle, 'IP Telephony' Deploying Voice -over-IP Protocols.pp 16 